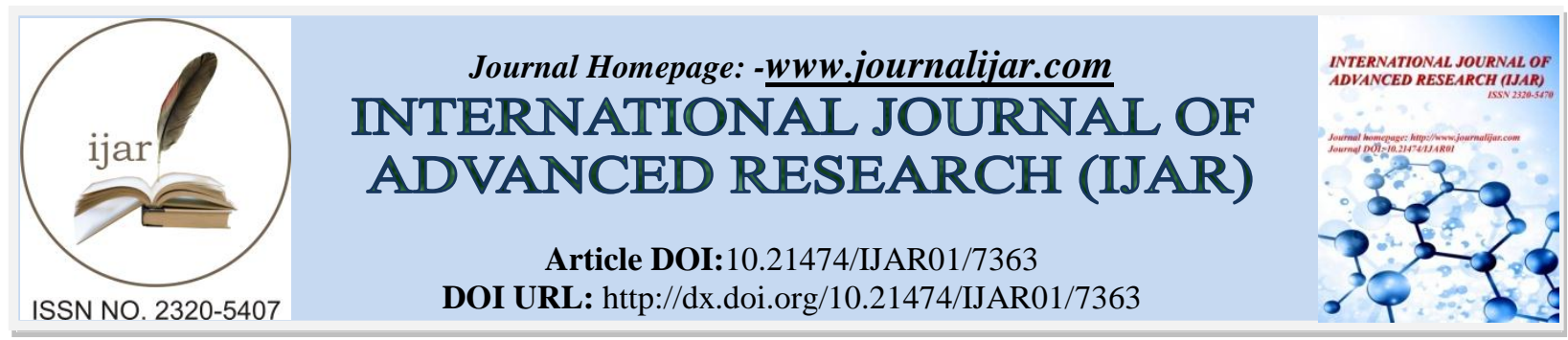

RESEARCH ARTICLE

\title{
THE PREDICTION OF COMPANY FINANCIAL PERFORMANCE BANKRUPTCY BY RATIO, STOCK AND Z-SCORE(PT MAIN TBK).
}

Putu Tirta Sari Ningsih and Rahayu.

University of MH. Thamrin, University of Prof. Dr. Moestopo (B) „Jakarta, Indonesia.

\section{Manuscript Info}

Manuscript History

Received: 07 May 2018

Final Accepted: 09 June 2018

Published: July 2018

Keywords:-

Financial repot, Ratio analysis, Stock analysis , Z-score.

\begin{abstract}
The objective of this study is to analyze The Predict of Company Financial Performance Bankruptcy By Ratio, Stock An Z-Score whether it is good or bad with PT Main TBK as the study object.

Based on the study objective, the hypotheses are : 1) Campany financial performance is good if it is measurement by using the ratio analysis tool; 2) Company financial performance is good if it measured uses stock analysis tool; 3) Company financial performance is good if it uses Z-score analysis tool.

The study design used was descriptive study, while the study method used was through documentation approach. The study sample is financial report from 2014 to 2017 of PT Main TBK. The sampling technique used area sampling (Sugiono,2009)). Analysis methods used were ratio analysis, stock analysis and $\mathrm{z}$-score analysis to answer the hypotheses.

The study result based on descriptive analysis toward variable used on company financial performance is proven to be good. The study result based based on the three analysis tools toward variable used on company financial performance is proven to be good. The three analysis result analysis toward the hypotheses test based on the empirical data of 3 hypotheses proven are the ratio analysis result toward company financial performance is proven to be good, the stock analysis toward company financial performance is proven to be good, and the $\mathrm{Z}$ score analysis result toward company financial performance is proven to be good.

Copy Right, IJAR, 2018,. All rights reserved.
\end{abstract}

\section{Introduction:-}

Online business rapidly grows nowadays. This global business has reached all over the world and one of this is retail online business. This business does not need huge investment . With just a little amount of money, this business can run well as long as the products suit the customers' taste or interest. Buyers do not need to come to see the stuff since they just need to use their cell phone to look for the items needed. The order is via online, then pay it by using e-money. Finally the order will be delivered to their front door. The industry that has the impact of the advanced technology is retailed industry such as Ramayana, Pasar Raya, Hypermart, Carefour etc that start fall apart ( Kompas newspaper, 2017). Meanwhile , the retail survived is PT Main TBK even though some branches have been closed down ( Pre-research) 
Bankruptcy is a major problem that needs to be wary about by every company . If the company is bankrupt it means the company is failed in running its business. Therefore, there must be an early analysis on bankruptcy prediction ( Demitras,2005)

Company ability to pay its debt, not only the short term but also the long term, company ability to provide working equity, company ability to run the business, company ability to make profit and pay devidend to the investors are the profile of healthy company. PT Main TBK becomes the object of the study where the researchers will predict the financial performance. Financial healthy performance will be analyzed throught ratio analysis,stock analysis and $\mathrm{z}$ score analysis

\section{Identification and Study Framework:-}

1. Is the financial performance condition of PT Main TBK good if it is measured by Ratio Analyisis

2. Is the financial performance condition of PT Main TBK good if it is measured by Stock Analyisis

3. Is the financial performance condition of PT Main TBK good if it is measured by Z-score

\section{Literature:-}

Company financial perdormance:-

Dimitras (2005) stated that company financial performance is an attainment achieved by the company that is stated in form of money value and described in a form of company financial report. Company financial performance can be measured by using ratio analysis,stock analysis and z-score analysis.

\section{Ratio Analysis:-}

Ratio analysis is an analysis tool used to measure the company financial performance from the liquidity, solvability, activity and profitability ( Athur et.al. , 2000)

\section{Stock Analysis:-}

According to Jacobson et.al. (2004) stock is a form of possession of company under the name of sameone in form of paper

Stock analysis is used to measure stock price and company condition with the measurement tools of earnings per share, price earning ratio, devidend per share, devidend yield and book value ( Jacobson et.al., 2004 )

\section{Z-score Analysis:-}

Z-score is an analysis tool of acceptable bankruptcy prediction and can be used to predict future possibility of a company image whether it is in normal or bankrupt condition. Zscore is current assets, current liability, working capital, total assets (A); earning before tax , total assets (B) ; earning before tax , current liability (C); sales, total assets (D), ( Altman , 1968 )

\section{Hypotheses:-}

Financial performance condition is measured by ratio analysis:-

Financial performance condition is good measured by ratio analysis

\section{Financial performance condition is measured by stock analysis:-}

Financial performance condition is good measured by stock analysis

Financial performance condition is measured by z-score analysis:-

Financial performance condition is good measured by z-score analysis

\section{Study Concept:-}

According to the study context, it creates study conceptual model The Prediction of Company Financial Performance Bankruptcy by Ratio, Stock and Z- score, and the analysis tools are ratio analysis, stock analysis and z-score with PT Main TBK as the study object Figure 1. 
Figure 1:-Conceptual Model

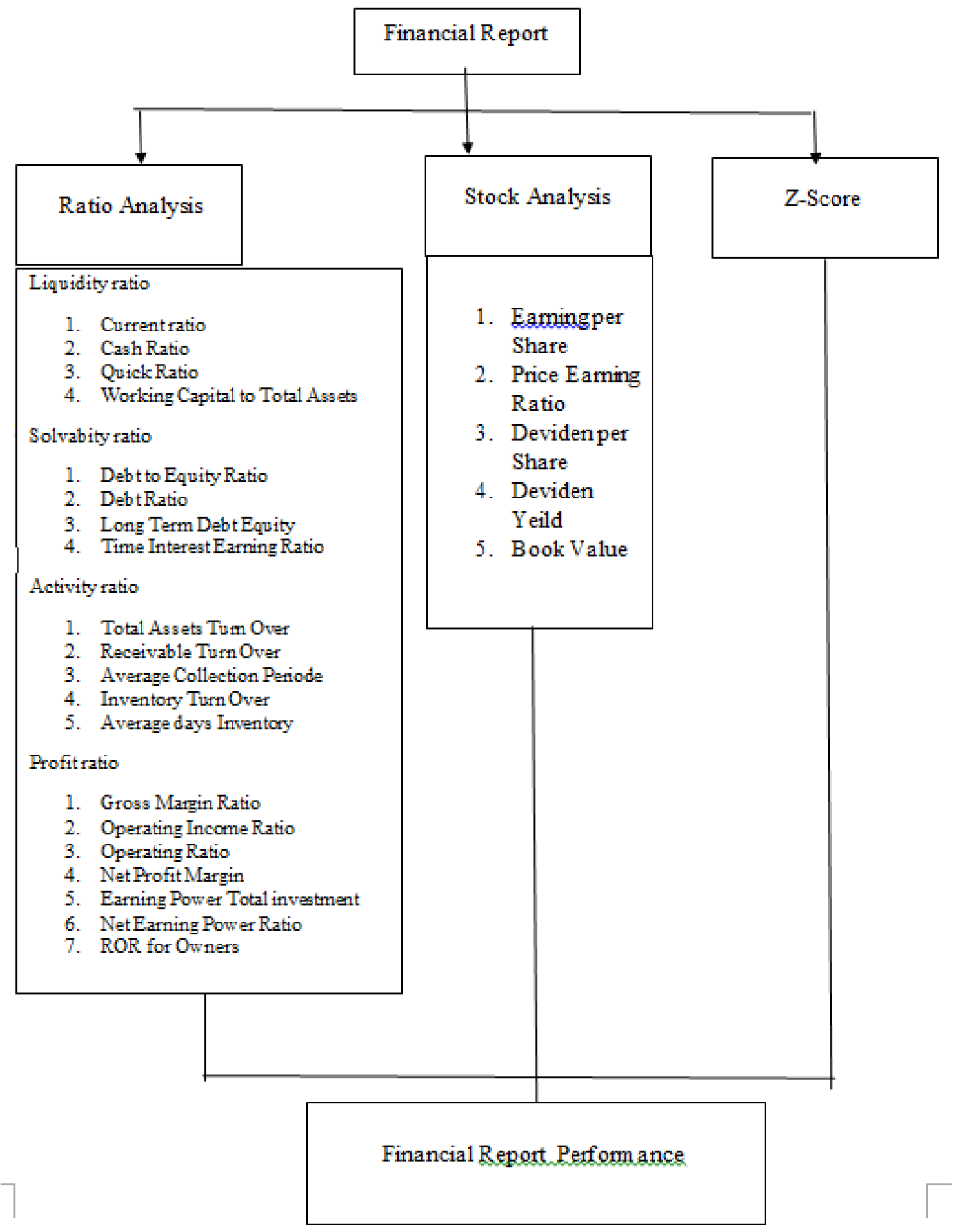

\section{Study Methodology:-}

The design of this study uses descriptive study through secondary data approach . the number of samples are 4 periods of financial report in PT Main TBK, stars from 2014 to 2017 in Jakarta , Indonesia. The sampling technique is area sampling (Sugiono, 2009) 
Analysis tools used to describe the study variables are by using ratio analysis, stock analysis and z-score.

\section{Research Result:-}

\section{Ratio analysis:-}

The result of liquidity ratio can be seen in Table 1.

Table 1:-Liquidity ratio ( Rp . million )

\begin{tabular}{|c|c|c|c|c|c|}
\hline & & 2014 & 2015 & 2016 & 2017 \\
\hline 1 & $\begin{array}{l}\text { Current Ratio } \\
\frac{\text { Current assets }}{\text { Current liability }}\end{array}$ & $\begin{array}{r}83 \% \\
\frac{2.111 .327}{2.518 .524}\end{array}$ & $\begin{array}{r}93 \% \\
2.272 .741 \\
2.439 .014\end{array}$ & $\begin{array}{r}115 \% \\
\frac{2.974 .000}{2.586 .354}\end{array}$ & $\begin{array}{r}113 \% \\
\frac{2.973 .745}{2.610 .824}\end{array}$ \\
\hline 2 & $\begin{array}{l}\text { Cash Ratio } \\
\frac{\text { Cash }}{\text { Current liability }}\end{array}$ & $\begin{array}{r}31 \% \\
\frac{785.895}{2.518 .521}\end{array}$ & $\begin{array}{r}39 \% \\
\quad 946.658 \\
2.439 .014\end{array}$ & $\begin{array}{r}66 \% \\
\frac{1.712 .844}{2.566 .354}\end{array}$ & $\begin{array}{r}61 \% \\
\frac{1.582 .817}{2.610 .824}\end{array}$ \\
\hline 3 & $\begin{array}{l}\text { Quick Ratio } \\
\frac{\text { Carent assets - Inventory }}{\text { Current liability }}\end{array}$ & $\begin{array}{c}46 \% \\
\frac{2.111 .517-}{\frac{955.811}{2.518 .521}}\end{array}$ & $\begin{array}{c}47 \% \\
\frac{2.272 .741-}{\frac{1.107 .811}{2.439 .014}}\end{array}$ & $\begin{array}{c}77 \% \\
\frac{2.974 .000-}{\frac{995.276}{2.566 .354}}\end{array}$ & $\begin{array}{c}75 \% \\
\frac{2.973 .745-}{1.005 .484} \\
\frac{2.610 .824}{}\end{array}$ \\
\hline 4 & $\begin{array}{l}\text { Working Capital to Total } \\
\text { Assets } \\
\frac{\text { Current assets -C. liabilities }}{\text { Total assets }}\end{array}$ & $\begin{array}{c}-16 \% \\
\frac{2.111 .507-}{2.518 .521} \\
2.421 .954\end{array}$ & $\frac{\frac{2.272 .741-}{2439.014}}{2.889 .291}$ & $\frac{\frac{2.974 .000-}{2.586 .3534}}{4.858 .878}$ & $\frac{\frac{2.973 .745-}{2.610 .824}}{5.427 .426}$ \\
\hline
\end{tabular}

The result of solvability ratio can be seen in Table 2 .

Table 2:-Solvability ratio ( Rp . million )

\begin{tabular}{|c|c|c|c|c|c|}
\hline & & 2014 & 2015 & 2016 & 2017 \\
\hline 1 & $\begin{array}{l}\text { Debt to Equity Ratio } \\
\frac{\text { Total liability }}{\text { Equity }}\end{array}$ & $\begin{array}{l}2108 \% \\
\frac{3.253 .691}{150.263}\end{array}$ & $\begin{array}{r}259 \% \\
\frac{2.783 .124}{1.106 .167}\end{array}$ & $\begin{array}{c}161 \% \\
\frac{3.003 .635}{1.855 .243}\end{array}$ & $\begin{array}{r}130 \% \\
\frac{3.099 .441}{2.377 .965}\end{array}$ \\
\hline 2 & $\begin{array}{l}\text { Debt Ratio } \\
\frac{\text { Total liability }}{\text { Total Assets }}\end{array}$ & $\begin{array}{c}95 \% \\
3.253 .691 \\
3.412 .954\end{array}$ & $\begin{array}{r}71 \% \\
2.783 .126 \\
3.889 .291\end{array}$ & $\begin{array}{c}62 \% \\
3.003 .635 \\
4.858 .878\end{array}$ & $\begin{array}{c}57 \% \\
\frac{3.099 .441}{5.427 .426}\end{array}$ \\
\hline 3 & $\begin{array}{l}\text { Long term Debt Equity } \\
\frac{\text { Long term liability }}{\text { Equity }}\end{array}$ & $\begin{array}{r}490 \% \\
\frac{735.170}{150.263}\end{array}$ & $\begin{array}{r}31 \% \\
344.110 \\
1.106 .167\end{array}$ & $\begin{array}{c}22 \% \\
\frac{415.281}{1.855 .243}\end{array}$ & $\begin{array}{c}20 \% \\
\frac{488.617}{2.377 .965}\end{array}$ \\
\hline 4 & $\begin{array}{l}\text { Time Interest Earning Ratio } \\
\text { EBIT }\end{array}$ & $11 \mathrm{X}$ & $30 X$ & $230 X$ & $159 \mathrm{X}$ \\
\hline
\end{tabular}




\begin{tabular}{|l|l|l|l|l|l|}
\hline & Interest of long term liability & $\frac{1.850 .594}{169.097}$ & $\frac{2.244 .621}{73.702}$ & $\frac{2.532 .666}{11.750}$ & $\frac{2.396 .300}{15.474}$ \\
\hline
\end{tabular}

The result of activity ratio can be seen in Table 3 .

Table 3:-Activity ratio ( Rp . million )

\begin{tabular}{|c|c|c|c|c|c|}
\hline & & 2014 & 2015 & 2016 & 2017 \\
\hline 1 & $\begin{array}{l}\text { Total assets turn over } \\
\underline{\text { Sales }} \text { Total assets }\end{array}$ & $\begin{array}{c}2.3 \mathrm{X} \\
\frac{7.925 .547}{3.412 .254}\end{array}$ & $\begin{array}{c}2.3 \mathrm{X} \\
\frac{9.006 .893}{3.889 .291}\end{array}$ & $\begin{array}{c}2 X \\
\frac{9.897 .046}{4.858 .878}\end{array}$ & $\begin{array}{c}1.8 \mathrm{X} \\
\frac{10.023 .961}{5.427 .426}\end{array}$ \\
\hline 2 & $\begin{array}{l}\text { Receivable turn over } \\
\frac{\text { Sales }}{\text { Average of receivable }}\end{array}$ & $\begin{array}{c}220 X \\
\frac{7.925 .547}{36.716}\end{array}$ & $\begin{array}{c}140 X \\
\frac{9.006 .893}{64.327}\end{array}$ & $\begin{array}{c}471 X \\
\frac{9.897 .046}{21.469}\end{array}$ & $\begin{array}{c}589 X \\
\frac{10.023 .961}{17.763}\end{array}$ \\
\hline 3 & $\begin{array}{l}\text { Average collection periode } \\
\frac{\text { Receivable X } 360}{\text { Sales }}\end{array}$ & $\begin{array}{c}1.66 \text { hari } \\
\frac{36.716 \times 360}{7.925 .547}\end{array}$ & $\begin{array}{c}2.57 \text { hari } \\
\frac{64.327 \times 360}{9.006 .893}\end{array}$ & $\begin{array}{c}0.78 \text { hari } \\
\frac{21.469 \times 360}{9.897 .046}\end{array}$ & $\begin{array}{c}0.63 \text { hari } \\
\frac{17.763 \times 360}{10.023 .961}\end{array}$ \\
\hline 4 & $\begin{array}{l}\text { Inventory turn over } \\
\frac{\text { Cost of goods }}{\text { Inventory }}\end{array}$ & $\frac{3.335 .638}{955.231}$ & $\frac{2.877 .507}{1.007 .811}$ & $\begin{array}{c}3.1 \mathrm{X} \\
\frac{3.085 .279}{995.276}\end{array}$ & $\begin{array}{c}3.7 \mathrm{X} \\
\frac{3.762 .021}{1.005 .484}\end{array}$ \\
\hline 5 & $\begin{array}{l}\text { Average days inventory } \\
\frac{\text { Inventory X } 360}{\text { Cost of goods }}\end{array}$ & $\begin{array}{c}103 \text { hari } \\
\frac{955.231 \times 360}{3.335 .638}\end{array}$ & $\begin{array}{c}126 \text { hari } \\
\frac{1.007 .811 \mathrm{X}}{2.8 \frac{360}{77.507}}\end{array}$ & $\begin{array}{c}116 \text { hari } \\
\frac{995.276 \times 360}{3.085 .279}\end{array}$ & $\begin{array}{c}98 \text { hari } \\
\frac{1.025 .484 \times 360}{3.762 .021}\end{array}$ \\
\hline
\end{tabular}

The result of profit ratio can be seen in Table 4 .

Table 4:-Profit ratio ( Rp . million )

\begin{tabular}{|c|c|c|c|c|c|}
\hline & & 2014 & 2015 & 2016 & 2017 \\
\hline 1 & $\begin{array}{l}\text { Gross margin ratio } \\
\frac{\text { Gross profit }}{\text { sales }}\end{array}$ & $\begin{array}{r}73 \% \\
\frac{5.818 .040}{7.925 .543}\end{array}$ & $\begin{array}{r}62 \% \\
\frac{5.671 .255}{9.006 .893}\end{array}$ & $\begin{array}{c}62 \% \\
6.211,767 \\
9.897 .046\end{array}$ & $\begin{array}{r}62 \% \\
\frac{6.261 .940}{10.023 .961}\end{array}$ \\
\hline 2 & $\begin{array}{l}\text { Operating income ratio } \\
\frac{\text { Operating income }}{\text { Sales }}\end{array}$ & $\begin{array}{r}23 \% \\
1.850 .544 \\
7.925 .543\end{array}$ & $\begin{array}{r}24 \% \\
2.244 .821 \\
9.006 .893\end{array}$ & $\begin{array}{c}35 \% \\
\frac{3.532 .666}{9.877 .046}\end{array}$ & $\begin{array}{c}23 \% \\
\frac{2.395 .300}{10.023 .961}\end{array}$ \\
\hline 3 & $\begin{array}{l}\text { Operating ratio } \\
\frac{\text { GGS + expenses }}{\text { Sales }}\end{array}$ & $\begin{array}{r}207 \% \\
2887.507+ \\
2.937 .013\end{array}$ & $\begin{array}{c}75 \% \\
\\
3.335 .638 \\
+3.341 .741+\end{array}$ & $\begin{array}{l}3.685 .279+ \\
3.683 .671+\end{array}$ & $\begin{array}{l}3.762 .021+ \\
3.852 .299+\end{array}$ \\
\hline
\end{tabular}




\begin{tabular}{|l|l|c|c|c|c|}
\hline & & $\frac{+255.951}{2.925 .593}$ & $\frac{123.660}{9.006 .893}$ & $\frac{31781}{9.877 .046}$ & $\frac{18.273}{10.023 .961}$ \\
\hline 4 & Net profit margin & $17 \%$ & $20 \%$ & $20 \%$ & $19 \%$ \\
& $\begin{array}{l}\text { Profit after tax } \\
\text { Sales }\end{array}$ & $\frac{1.419 .116}{7.925 .543}$ & $\frac{1.780 .848}{9.006 .893}$ & $\frac{2.019 .705}{9.877 .040}$ & $\frac{1.907 .077}{10.023 .960}$ \\
\hline 5 & $\begin{array}{l}\text { Earning power total } \\
\text { invesment }\end{array}$ & $54 \%$ & $57 \%$ & $68 \%$ & $44 \%$ \\
& $\begin{array}{l}\text { Earning before tax } \\
\text { Total Assset }\end{array}$ & $\frac{1.850 .546}{3.421 .954}$ & $\frac{2.244 .821}{3.899 .291}$ & $\frac{3.352 .666}{4.858 .878}$ & $\frac{2.395 .300}{5.427 .421}$ \\
\hline 6 & $\begin{array}{l}\text { Net earning power ratio } \\
\text { Earning after tax }\end{array}$ & $\frac{1.419 .116}{3.421 .954}$ & $\frac{1.780 .848}{3.899 .291}$ & $\frac{2.019 .705}{4.858 .878}$ & $\frac{1.907 .077}{5.427 .421}$ \\
\hline 7 & $\begin{array}{l}\text { Total asets } \\
\text { ROR for owners }\end{array}$ & $\frac{1.419 .116}{150 \%}$ & $\frac{1.780 .848}{1.106 .167}$ & $\frac{2.019 .705}{1.855 .243}$ & $\frac{1.907 .077}{2.377 .965}$ \\
\hline
\end{tabular}

\section{Stock analyis:-}

The result of stock analysis can be seen in Table 5 .

Table 5:-Stock analysis ( Rp . million )

\begin{tabular}{|c|c|c|c|c|c|}
\hline & & 2014 & 2015 & 2016 & 2017 \\
\hline 1 & $\begin{array}{l}\text { Earning per share } \\
\frac{\text { Earning after tax }}{\text { Number of shares }}\end{array}$ & $\begin{array}{l}486.498 \\
\frac{1.419 .116}{2.917 .} \\
\end{array}$ & $\begin{array}{l}610.506 \\
\frac{1.780 .848}{2.917} \\
\end{array}$ & $\begin{array}{l}692.391 \\
\frac{2.019 .905}{2.917 .} \\
\end{array}$ & $\begin{array}{r}653.780 \\
\frac{1.907 .077}{2.917} \\
\end{array}$ \\
\hline 2 & $\begin{array}{l}\text { Price earning ratio } \\
\frac{\text { Stock market price }}{\text { Earning per share }}\end{array}$ & $\begin{array}{l}112 \% \\
\underline{5.450} \\
486.408 \\
\end{array}$ & $\begin{array}{l}89 \% \\
\underline{5.450} \\
610.506 \\
\end{array}$ & $\begin{array}{l}78 \% \\
\underline{5.450} \\
692.391 \\
\end{array}$ & $\begin{array}{l}83 \% \\
\underline{5.450} \\
653.780 \\
\end{array}$ \\
\hline 3 & $\begin{array}{l}\text { Deviden per share } \\
\text { Deviden payment by cash } \\
\text { Number of shares } \\
\text { outstanding }\end{array}$ & $\begin{array}{c}164 \\
\frac{480.448}{2.917}\end{array}$ & $\begin{array}{l}291 \\
\frac{851.448}{2.917}\end{array}$ & $\begin{array}{c}427 \\
\frac{1.246 .826}{2.917}\end{array}$ & $\begin{array}{c}491 \\
\frac{1.434 .023}{2.917}\end{array}$ \\
\hline 4 & $\begin{array}{l}\text { Deviden Yeild } \\
\frac{\text { Deviden per share }}{\text { Stock market price }}\end{array}$ & $\begin{array}{l}3 \% \\
\frac{164}{5.450}\end{array}$ & $\begin{array}{l}5 \% \\
\frac{291}{5.450}\end{array}$ & $\begin{array}{l}7 \% \\
\frac{427}{5.450}\end{array}$ & $\begin{array}{l}9 \% \\
\underline{491} \\
5.450\end{array}$ \\
\hline 5 & $\begin{array}{l}\text { Book value } \\
\text { Owner's equity } \\
\begin{array}{l}\text { Number of shares } \\
\text { outstanding }\end{array}\end{array}$ & $\begin{array}{l}51.478 \\
\frac{150.163}{2.917}\end{array}$ & $\begin{array}{l}379.213 \\
\frac{1.106 .167}{2.917}\end{array}$ & $\begin{array}{c}636.010 \\
\frac{1.855 .243}{2.917}\end{array}$ & $\begin{array}{r}815.209 \\
\frac{2.377 .965}{2.917}\end{array}$ \\
\hline
\end{tabular}

\section{Z-score:-}

The result of Z-score analysis can be seen in Table 6. 
Table 6:-Z- score ( Rp . million )

\begin{tabular}{|c|c|c|c|c|}
\hline & 2014 & 2015 & 2016 & 2017 \\
\hline Current Assets & 2.111 .327 & 2.272 .741 & 2.974 .000 & 2.973 .745 \\
\hline Current liability & 2.518 .521 & 2.439 .014 & 2.566 .354 & 2.610 .824 \\
\hline Working Capital (CA- CL) & $(407.014)$ & $(166.273)$ & 407.651 & 362.921 \\
\hline Total Assets & 3.421 .954 & 3.889 .291 & 4.858 .878 & 5.427 .426 \\
\hline $\mathrm{A}(\mathrm{WC}: \mathrm{TA})$ & -0.11 & -0.04 & 0.08 & 0.66 \\
\hline Earning before tax & 1.850 .546 & 2.244 .821 & 3.532 .666 & 2.395 .300 \\
\hline Total Assets & 3.421 .954 & 3.889 .291 & 4.858 .878 & 5.427 .426 \\
\hline $\mathrm{B}(\mathrm{E}: \mathrm{TA})$ & 0.54 & 0.57 & 0.72 & 0.44 \\
\hline Earning before tax & 1.850 .546 & 2.244 .821 & 3.532 .666 & 2.395 .300 \\
\hline Current Liability & 2.518 .521 & 2.439 .014 & 2.566 .354 & 2.610 .824 \\
\hline $\mathrm{C}(\mathrm{E}: \mathrm{CL})$ & 0.73 & 0.92 & 1.97 & 0.91 \\
\hline Sales & 7.925 .453 & 9.006 .893 & 9.877 .048 & 10.023 .967 \\
\hline Total Assets & 3.421 .954 & 3.889 .291 & 4.858 .878 & 5.427 .426 \\
\hline $\mathrm{D}$ (S: TA) & 2.31 & 2.31 & 2.03 & 1.84 \\
\hline $\mathrm{Z}$-Score $(\mathrm{A}+\mathrm{B}+\mathrm{C}+\mathrm{D})$ & 3.67 & 3.84 & 4.2 & 3.25 \\
\hline $\begin{array}{l}\text { Company condition } \\
\text { Z -score }>0.862\end{array}$ & $\begin{array}{c}3.67>0.862 \\
\text { Good }\end{array}$ & $\begin{array}{l}1.84>0.862 \\
\text { Good }\end{array}$ & $\begin{array}{c}4.2>0.862 \\
\text { Good }\end{array}$ & $\begin{array}{l}3.25>0.862 \\
\text { Good }\end{array}$ \\
\hline
\end{tabular}

Result and Discussion:-

Ratio analysis value

Liquidity ratio analysis:-

Campany liquidity is good when a company is able to fulfil its short -term debt with current assets as seen in

Table 7.

Table7:-Liquidity ratio

\begin{tabular}{|l|l|l|l|l|l|r|}
\hline & $\mathbf{2 0 1 4}$ & $\mathbf{2 0 1 5}$ & $\mathbf{2 0 1 6}$ & $\mathbf{2 0 1 7}$ & Condition \\
\hline 1 & Current ratio & $83 \%$ & $93 \%$ & $115 \%$ & $113 \%$ & Good \\
\hline 2 & Cash ratio & $31 \%$ & $39 \%$ & $66 \%$ & $61 \%$ & Good \\
\hline 3 & Quick ratio & $46 \%$ & $47 \%$ & $77 \%$ & $75 \%$ & Good \\
\hline 4 & Working capital ratio & $-16 \%$ & $5 \%$ & $7 \%$ & $6 \%$ & Good \\
\hline
\end{tabular}

\section{Current Ratio:-}

The ability of current assets to pay for the current liability from 2014 to 2016 increased . In 2017 current ratio of $113 \%$ where current liability $\mathrm{Rp} \mathrm{1,-}$ guaranteed by current assets of Rp.1,3 with meant the company is still able to fully pay current liability by using current assets as seen in Table 7.

\section{Cash Ratio:-}

Measuring cash ability in paying for the current liability in Table 7 from year to year has increased . In 2017 current liability $\mathrm{Rp} 1,-$ is guaranteed by cash $\mathrm{Rp} 0.61$. 


\section{Quick ratio:-}

The ability of current assets decreased by stock to pay for current liability . In the Table 7 it has increase in 2017 of $75 \%$ not too different with the previous year.

\section{Working capital ratio:-}

Working capital ratio achived from total assets used for company operasional . In Table 7 working equity to total assets is still minus, and started from 2015 to 2017 it was getting better.

Analisa Ratio Solfabilitas:-

Company solvability is assumed good if company has adequent assets to pay for all of its debts as seen in Table 8.

Table 8.:-Ratio Solfabilitas

\begin{tabular}{|r|r|r|r|r|r|r|}
\hline & & $\mathbf{2 0 1 4}$ & $\mathbf{2 0 1 5}$ & $\mathbf{2 0 1 5 d e b t}$ & $\mathbf{2 0 1 7}$ & Condition \\
\hline 1 & Debt to equty ratio & $2108 \%$ & $259 \%$ & $161 \%$ & $130 \%$ & Good \\
\hline 2 & Debt ratio & $95 \%$ & $71 \%$ & $62 \%$ & $57 \%$ & Good \\
\hline 3 & Long term debt equity & $490 \%$ & $31 \%$ & $22 \%$ & $20 \%$ & Good \\
\hline 4 & Time interest earning ratio & $11 \mathrm{X}$ & $30 \mathrm{X}$ & $230 \mathrm{X}$ & $159 \mathrm{X}$ & Good \\
\hline
\end{tabular}

\section{Debt to Equity Ratio:-}

Company fulfils its short-term and long-term debt by using its own equity. In Table 8 , debt to equity ratio from year to year has decreased which meant the equty increased and debt decreased.

\section{Debt Ratio:-}

Company fulfils its short-term and long-term debt by using its total assets. In Table 8 debt ratio in 2014 to 2017 decreased which meant the company increased total assets more and decreased debt

\section{Long term Debt Equity:-}

Company in long-term debt is paid by using its own equity as seen in Table 8 long termdebt equity trend decreased . In 2017 it was $18 \%$ which meant the company slightly depended on debt but dependent more its own equity.

\section{Time Interest Earning Ratio:-}

Time needed by the company to fully pay for its debt by using company earning is quite good as seen in Table 8 where time interest earning ratio in 2014 to 2016 increased. Then, in 2017 it was $159 \mathrm{X}$ with a little decrease compared with 2016

\section{Activity ratio:-}

Company activity ratio is assumed good where the company shows its effectiveness in using assets possessed

Table 9:-Activity ratio

\begin{tabular}{|l|l|l|l|l|l|l|}
\hline & & $\mathbf{2 0 1 4}$ & $\mathbf{2 0 1 5}$ & $\mathbf{2 0 1 6}$ & $\mathbf{2 0 1 7}$ & Condition \\
\hline 1 & Total assests turn over & $2.3 \mathrm{X}$ & $2.3 \mathrm{X}$ & $2 \mathrm{X}$ & $1.8 \mathrm{X}$ & Ugly \\
\hline 2 & Receivable tur over & $220 \mathrm{X}$ & $140 \mathrm{X}$ & $471 \mathrm{X}$ & $589 \mathrm{X}$ & Good \\
\hline 3 & Average collegtion periode & 1.66 days & 2.57 days & 0.78 days & 0.63 days & Good \\
\hline 4 & Inventory turn over & $3.5 \mathrm{X}$ & $2.8 \mathrm{X}$ & $3.1 \mathrm{X}$ & $3.7 \mathrm{X}$ & Good \\
\hline 5 & Average days inventory & 103 days & 126 days & 116 days & 98 days & Good \\
\hline
\end{tabular}

Total assets turn over:-

Company assets turn over was not effective and efficient. In the Table 9, in 2014 to 2017 it decreased which meant sale to total assets increase has not been followed by total assets turn over .

\section{Receivable turn over:-}

Receivable turn over is measuring how many times receivable turn over to become cash in 1 year as seen in Table 9 from 2014 to 2017 it has increase which last year it was $589 \mathrm{X}$. 
Average collection periode:-

Receivable average periode is < 30 days, as seen in Table 9, in2014 to 2017 it showed receivable average period $<30$ days (good).

\section{Inventory turn over:-}

Inventory turn over ran effectively as seen in Table 9 in 2015 to 2017 increased which means the increase of good main proce is followed by increase in inventory

\section{Average days inventory:-}

Average days inventory is adequately good which is the average day of inventory becomes good stock year to year decreased . it meant inventory did not last long as stock. Inventory was used for goods and sold goods as seen in Table 9

Profit Ratio:-

Profitability ratio of the company in making profit in certain period is quite good as seen in Table $\mathbf{1 0 .}$

Table10:-Profit Ratio

\begin{tabular}{|l|l|l|l|l|l|l|}
\hline & & $\mathbf{2 0 1 4}$ & $\mathbf{2 0 1 5}$ & $\mathbf{2 0 1 6}$ & $\mathbf{2 0 1 7}$ & Condition \\
\hline 1 & Gross margin ratio & $6.9 \%$ & $62 \%$ & $62 \%$ & $63 \%$ & Good \\
\hline 2 & Operating income ratio & $23 \%$ & $24 \%$ & $35 \%$ & $23 \%$ & Good \\
\hline 3 & Operating ratio & $76 \%$ & $75 \%$ & $74 \%$ & $76 \%$ & Good \\
\hline 4 & Net profit margin & $17 \%$ & $20 \%$ & $20 \%$ & $19 \%$ & Good \\
\hline 5 & $\begin{array}{l}\text { Earning power total } \\
\text { investment }\end{array}$ & $54 \%$ & $57 \%$ & $72 \%$ & $44 \%$ & Good \\
\hline 6 & Net earning power ratio & $41 \%$ & $46 \%$ & $41 \%$ & $35 \%$ & Good \\
\hline 7 & ROR for owners & $946 \%$ & $160 \%$ & $108 \%$ & $80 \%$ & Good \\
\hline
\end{tabular}

Gross margin ratio:-

In Table 10 company ability in having gross profit through sale activity from year to year has been increasing

Operating income ratio:-

Company operating income ratio ran well from 2015 to 2017 and keep growing as seen in Table 10

\section{Operating ratio:-}

Measuring main price and operasional expense compared with selling activity from year to year is quate stable with operating ratio between $74 \%$ and $76 \%$ as seen in Table 10 Table 10

Net profit margin:-

In Table 10 Company net profit after taxes toward sale is between $17 \%$ and $19 \%$ the change is not significant which meant the company is still able to make net profit through sale .

\section{Earning power total investment:-}

The level of assets return through net profit before taxes in 2014 to 2016 increased. In 2017 earning power total assets decreased sharply to be $44 \%$, however, this level is still considered good enough as seen in Table 10

\section{Net earning power ratio;-}

The level of assets return through net profit after taxes in 2014 to 2016 increased . In 2017 net earning power ratio slightly decreased to $35 \%$, this level is still considered good enough as seen in Table $\mathbf{1 0 .}$

\section{ROR for orners:-}

Profit after taxes compared with owner equity in 2014 to 2016 increased . In 2017 ROR for Owners slightly decreased to $80 \%$, however, it is still consider good enough as seen in Table $\mathbf{1 0 .}$ 
Stock analysis:-

Sock analysis measures company ability in making stocks interesting for the investors is assumed really good as seen in Table 11.

Table 11:-Stock analysis

\begin{tabular}{|r|r|c|c|c|c|r|}
\hline & & $\mathbf{2 0 1 4}$ & $\mathbf{2 0 1 5}$ & $\mathbf{2 0 1 6}$ & $\mathbf{2 0 1 7}$ & Condition \\
\hline 1 & Earning per share & 486.498 & 610.506 & 692.391 & 653.780 & Good \\
\hline 2 & Price earning ratio & $112 \%$ & $89 \%$ & $78 \%$ & $83 \%$ & Good \\
\hline 3 & Deviden per share & 164 & 291 & 427 & 491 & Good \\
\hline 4 & Deviden yeild & $3 \%$ & $5 \%$ & $7 \%$ & $9 \%$ & Good \\
\hline 5 & Book value & 51.478 & 379.213 & 636.010 & 815.209 & Good \\
\hline
\end{tabular}

Earning per share:-

Measuring company ability in making earnings per share as seen in Table 11, earning per share in 2014 to 2016 increased . In 2017 it was slightly decreased which meant the company is still able to earn profit.

\section{Price earning ratio:-}

Price earning ratio describes company earning toward stock price that shows the return is decrease and increases, the company condition is good that price pershare stable can be seen in Table 11.

\section{Devidend per share;-}

Deviden per share measures dividend return level given by company to the stock holder from 2014 to 2017 increased . In Table 11 , in 2017 company was able to give dividend of Rp.514.226 per share distributed

Devidend yield:-

In Table 11 dedivend yield measures dividend yield level given by each share compared with stock market price distributed shows increasing. In the last 2017 it showed devidend yield 9,1\% .

\section{Book value:-}

Owner equity compared with distributed stock number increases every year which means equity increase is also followed by the increase number of share distributed as seen in Table 11.

\section{Z-score:-}

A good Z-score analysis of financial performance is > 0.862 as seen in Table 12.

Table 12:-Z- score analysis

\begin{tabular}{|c|c|c|c|c|}
\hline & 2014 & 2015 & 2016 & 2017 \\
\hline Current assets & 2.111 .327 & 2.272 .741 & 2.974 .000 & 2.973 .745 \\
\hline Current liability & 2.518 .521 & 2.439 .014 & 2.566 .354 & 2.610 .824 \\
\hline Working Capital & $(407.014)$ & $(166.273)$ & 407.651 & 362.921 \\
\hline Total assets & 3.421 .954 & 3.889 .291 & 4.858 .878 & 5.427 .426 \\
\hline $\mathrm{A}$ & -0.11 & -0.04 & 0.08 & 0.66 \\
\hline Ebit & 1.850 .546 & 2.244 .821 & 3.532 .666 & 2.395 .300 \\
\hline Total assets & 3.421 .954 & 3.889 .291 & 4.858 .878 & 5.427 .426 \\
\hline $\mathrm{B}$ & 0.54 & 0.57 & 0.72 & 0.44 \\
\hline Ebit & 1.850 .546 & 2.244 .821 & 3.532 .666 & 2.395 .300 \\
\hline Current liability & 2.518 .521 & 2.439 .014 & 2.566 .354 & 2.610 .824 \\
\hline $\mathrm{C}$ & 0.73 & 0.92 & 1.97 & 0.91 \\
\hline Sales & 7.925 .453 & 9.006 .893 & 9.877 .048 & 10.023 .967 \\
\hline Total assets & 3.421 .954 & 3.889 .291 & 4.858 .878 & 5.427 .426 \\
\hline $\mathrm{D}$ & 2.31 & 2.31 & 2.03 & 1.84 \\
\hline
\end{tabular}




\begin{tabular}{|l|c|c|c|c|}
\hline & & & & \\
\hline Z -Score $(\mathrm{A}+\mathrm{B}+\mathrm{C}+\mathrm{D})$ & 3.67 & 3.84 & 4.2 & 3.25 \\
\hline & & & & \\
\hline Condition & Good & Good & Good & Good \\
\hline
\end{tabular}

The analysis result of $\mathrm{z}$-score in Table 12 of financial performance condition from 2014 to 2017 where the $\mathrm{z}$ score in this table is $>0,862$, which means financial performance condition the company is good

The Conclusion of this study is hypotheses test based on empirical data is proven tobe good. There are three that are proven with are the ratio analysis result toward company financial performance is proven to be good, the stock analysis toward company financial performance is proven to be good, and the $\mathrm{Z}$ score analysis result toward company financial performance is proven to be good.

\section{Reference:-}

1. Altman E.I. (1968). Financial ratios discriminant analysis and the prediction of corporate bankruptcy. The Journal of Finance 23. 589-609.

2. Altman. Edward I.. R.G. Haldeman. and P. Narayanan. "Zeta-analysis. A New Model. Aziz, A., D. Emanuel and G. Lawson. 1988. Bankruptcy prediction - An investigation of cash flow based models.

3. Arthur J.K et.al. ( 2000)Basic Management Pearson Education Asia Pte Ltd

4. Beaver. W. H. (1967). Financial Ratios as Predictors of Failure. Empirical Research in Accounting: Selected

5. Becchetti, L. and Sierra J., "Bankruptcy risk a nd productive efficiency in manufacturing firms", Journal of Banking and Finance, Vol. 27, 2002.

6. Bilderbeek, J., "An empirical study of the predictive ability of financial ratios in the Netherlands", Zeitschrift Für Betriebswirtschaft May 1979.

7. Callahan; K. R; Stetz, G. S; Brooks, L. M (2007). Project Management Accounting: Budgeting, Tracking, and Reporting Costs and Profitability. Published by John Willey and Sons, Inc, Hoboken, New Jersey.

8. Courtis. J.K. (1978). Modeling a financial ratios categorical framework. Journal of Business Finance and Accounting. 5. 371-386.

9. Dimitras, A.I., Zanakis, S.H., Zopounidis, C. (2005 ). A survey of business failures with an emphasis on prediction methods and industrial applications. European Journal of Operational Research,

10. Edmister. R.O. (1972). An empirical test of financial ratio analysis for small business failure prediction. The Journal of Financial and Quantitative Analysis. 7. 1477-1493.

11. Eisenbeis, R. A., (1977), "Pitfalls in the Application of Discriminant Analysis in Business, Finance, and Economics,"

12. Gentry, J.A., Newbold P. and Whitford D.T., "C lassifying bankrupt firms with funds flow components", Journal of Accounting Research, Vol. 23, nr. 1, 1985

13. Gesternberg. (20015) Financial Organizational and Management of Business. Printice -Hall, Inc., Englewood Cliffs , New Jersey, Maruzen Company, Ltd., Tokyo

14. Jacobson et.al. (2004) "Credit risk versus Capital Requirement under Basell II : Are SME Loans and Retail Different " Journal of financial services Research, forthcoming .

15. Howard (2007), Working Capital, Its Management and Control, Macdonald \& Evans , Ltd., John Street, London W.C.

16. Keown, Martin, Petty and Scott,JR (2002) Financial Management Principles and Application, by Ptintice Hall

17. Keasey, K. and R. Watson. 1986. The prediction of small company failure: Some behavioral evidence for the UK.

18. Kompas (2017) newspaper, Jakarta,Indonesia

19. Laitinen. E.K. (1992). Prediction of failure of a newly founded firm. Journal of Business Venturing. 7. 323-340

20. Shahzad Ijaz, UIMS-PMAS-Arid, (2013) Assessing the Financial Failure Using Z-Score and Current Ratio

21. Sugiono (2009). Metode Penelitian Bisnis, 3 th ed. Bandung. Alfabeta. 